\title{
Computer Simulation of C-N-V Precipitates Evolution Based on Local Concentration Fluctuations
}

\author{
J. Aldazabal ${ }^{1, a}$, C. Garcia-Mateo ${ }^{2, b}$ \\ ${ }^{1}$ CEIT and Tecnun (University of Navarra) \\ Paseo Manuel Lardizábal 15, 20018 San Sebastián, Spain \\ 2 Department of Physical Metallurgy, Centro Nacional de Investigaciones \\ Metalúrgicas (CENIM-CSIC), Avda. Gregorio de Amo 8, E-28040 Madrid, Spain \\ a jaldazabal@ceit.es, b cgm@cenim.csic.es
}

Keywords: Microstructural evolution, Ostwald ripening, diffusion, precipitates, computer simulation.

\begin{abstract}
Abstact. From a "macroscopic" point of view, steel composition is assumed to vary smoothly along its microstructure. A closer look reveals that, on the atomic level the material composition does not change so smoothly. Single atoms jump randomly along the crystal lattice due to their thermal energy. These random jumps create sporadic zones of the crystal with higher concentration of certain species, and they are responsible for many phenomena, such as precipitation, Ostwald ripening, some phase transformations... This paper proposes a model to simulate the evolution of $\mathrm{C}-\mathrm{N}-\mathrm{V}$ precipitates in microalloyed steels heat treated in the range of warm temperatures (800-900 ${ }^{\circ} \mathrm{C}$ ); when the matrix is austenite (fcc), thus taking into account for the local composition fluctuations. The model works by dividing the space into very small cells, containing a single atomic cell each. If during the random movement of atoms a cell that touches a precipitate reaches some critical composition, it is very easy to stick it to the precipitate by changing its "phase". But it is also possible that some atoms escape from the precipitate by jumping to the austenitic matrix. Both processes happening simultaneously, and which one is leading depends on the atoms energy, i.e. system temperature.
\end{abstract}

\section{Introduction}

The mechanical properties of steels, such as elastic limit or yield stress, have been notably improved during the last decades. This increase was obtained in many cases as the result of adding small amounts of certain substances into the steel. Some substances, such as C, N, Nb or V, help to get small precipitates into the steel microstructure. These precipitates are very hard, and the dislocations, that move along the material during the plastic deformation, are not able to cross them. This results on pining dislocation, what increases the elastic limit. Even with the same volume fraction of precipitates it is possible to get different elastics limits, thus, many small precipitates distributed into the matrix are more effective pinning dislocations than a few big ones [1].

Using heat treatments it is possible to change the sizes and distribution of precipitates present, changing the resulting mechanical properties.

On the other hand, if a material with precipitates within its microstructure is held it at enough temperature, another phenomena could start, the Ostwald ripening effect. This phenomenon changes the precipitates sizes distribution without changing its volume fraction, by increasing the size of big particles and shrinking the small ones [2]. The reason for this effect is that at enough high temperatures some atoms at the surface of precipitates have enough thermal energy to jump into the surrounding matrix, and some other atoms of the matrix get "stick" into precipitates surface resulting in a dissolving-reprecipitating phenomenon. 
This paper proposes a model to study the evolution of $\mathrm{C}-\mathrm{N}-\mathrm{V}$ present in a $\mathrm{V}$ microalloyed steels treated in the range of warm temperatures $\left(800-900{ }^{\circ} \mathrm{C}\right)$. This model takes into account the jump of the $\mathrm{V}$ atoms from the precipitates into the matrix, its diffusion though the austenitic matrix and its precipitation into the $\mathrm{C}-\mathrm{N}-\mathrm{V}$ particles.

\section{Model description}

The purpose of the model is to simulate the evolution of the $\mathrm{C}-\mathrm{N}-\mathrm{V}$ precipitates at warm temperatures on a V microalloyed steel. To simulate this evolution, the first step is to introduce the initial microstructure in the model. After defining the microstructure the model simultaneously diffuses $\mathrm{V}$ atoms through the austenitic matrix, "evaporating" some atoms from, "condensating" other atoms at, the precipitates surface. As $\mathrm{V}$ atom diffuses several orders of magnitude slower that the $\mathrm{N}$ or $\mathrm{C}$ atoms [3,4], the model proposed in this paper will assume that $\mathrm{N}$ and $\mathrm{C}$ diffuses infinitely fast, i.e. the matrix and precipitates composition is homogeneous in terms of $\mathrm{C}$ and $\mathrm{N}$. Finally the model gives the description of the microstructure in terms of phases and elements distribution. Due to the high computation power required to run the model only two-dimensional simulations were performed for this paper, and further work is required to extend it to the threedimensional case.

The following subsections offer a detailed stepped explanation in how the model works.

Initial microstructure generation. The first step to simulate the evolution of the microstructure is to introduce it into the model. The topological description of the microstructure is discretized into small cubic cells (hereafter called voxels). It is known that both phases, austenite and precipitates, have an fcc structure as shown in Fig. 1a, where dark atoms are in the $\left(\begin{array}{lll}0 & 0 & 1 / 2\end{array}\right)$ atomic planes. The volume of each voxel is the same as the volume of a crystallographic cell, i.e. for warm range temperatures a lattice parameter of about $0.364 \mathrm{~nm}$. A crystallographic cell has some atoms that are shared by two cells (face atoms) and other by eight neighbour cells (vertex atoms). From the logical point of view it is more interesting that atoms belong to a single voxel. It is possible to obtain this just by displacing [ $\left[1 / 4{ }^{1 / 4}{ }^{1 / 4}\right]$ a "box" that corresponds to a crystallographic cell, i.e. $1 / 4^{\text {th }}$ of the lattice parameter in the three crystallographic axes. Fig. 1b shows a top view of the Fig.1a, where the dashed lines define the volume of the voxel as previously defined. Thus defined, the voxel contains four atoms not shared with any neighbour.

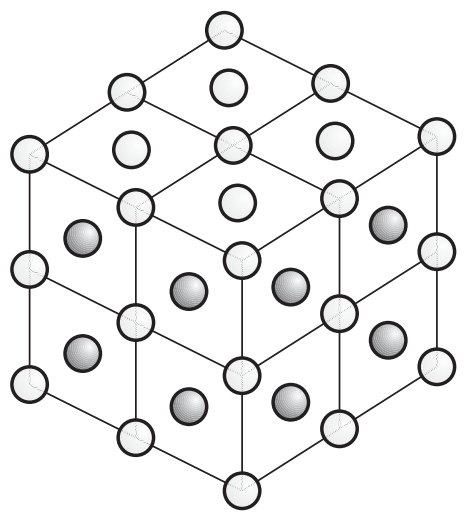

(a)

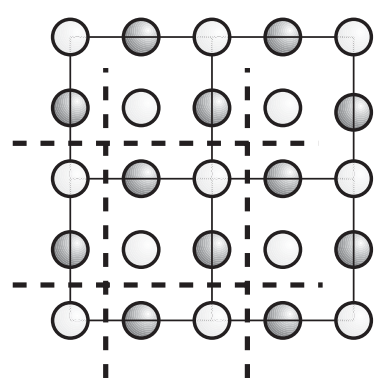

(b)

Figure 1: (a) fcc cell where dark spheres corresponds to atoms located in the $\left(\begin{array}{lll}0 & 0 & 1 / 2\end{array}\right)$ planes.

(b) Top view of the previous figure where dashed lines delineate the voxel used in the model.

Once the voxels are defined the initial microstructure has to be discretized into these voxels. In this work, initial microstructure was defined locating non-touching spherical particles into an austenitic matrix. To avoid the side effects during the simulation it is interesting to use periodical boundary conditions, not only in the diffusion but also in the initial microstructure generation [5]. Using these conditions, when an atom tries to escape from the microstructure crossing a side, it will 
appear in the opposite side. The same happens when locating initial particles, if a portion of a precipitate is placed outside the microstructure this portion will be re-located inside but at the opposite side.

It also assumed that $\mathrm{Fe}$ and $\mathrm{V}$ are substitutional elements while $\mathrm{C}$ and $\mathrm{N}$ are interstitial. According to this hypothesis the model accepts four substitutional and four interstitial atoms per voxels, corresponding to lattice sites and to octahedral interstices [6]. When all voxels are assigned to different phases they are filled with atoms. From a pre-assigned composition to each phase, and knowing the fraction of voxels that correspond to matrix and precipitates, it is possible to calculate the number of atoms that correspond to each phase. Different kinds of atoms are located in each phase randomly, allowing local composition fluctuations but maintaining the average pre-fixed composition of each phase. Each atom of the microstructure has an associated value to know if it is "movable", when it is in the matrix, or "fixed", when it is in a precipitate or stick to its surface. When generating the initial microstructure every atom located in the matrix is "movable" and every atom located in the precipitates is "fixed".

Phase assignation. As previously explained, during the initial discretization process the voxels are assigned to matrix or to precipitates. From the point of view of the model, it is very important to know which matrix voxels are in contact with precipitates, meaning that they share a face or an edge with a precipitate voxel. These "special" voxels that belongs to the matrix, and initially has same average composition, are defined as interface voxels. During the simulation these interface voxels will have some special characteristics, being the only matrix voxels that could swap their state from "matrix" to "precipitate".

During the simulation, due to the diffusion of atoms, the elements concentration in the voxels fluctuates. As it was mentioned, $\mathrm{V}$ is going limit the microstructural evolution, due to its low diffusion rate. The two phases considered by the model are the austenite (nearly all substitutional sites filled by $\mathrm{Fe}$ ) and $\mathrm{C}-\mathrm{N}-\mathrm{V}$ precipitates (almost substitutional sites filled by $\mathrm{V}$ ). If during the simulation, a voxel get rich in Fe, or it has more Fe that $\mathrm{V}$ it will be assigned to austenite. On the other hand, if an "interface voxel" has more $\mathrm{V}$ than Fe it will be assigned to a precipitate. If a voxel has the same number of $\mathrm{Fe}$ and $\mathrm{V}$ atoms it will maintain its previous state.

Diffusion algorithm. From the macroscopic point of view the diffusion process takes place due to the gradients on composition, when trying to homogenise them. From a very small scale, atoms, due to their thermal energy, are able to jump from one lattice site to other sites, swapping with neighbours or by swapping with vacancies, i.e. with void in the lattice. Due to these random jumps the composition of atomic cells fluctuates. The macroscopic effect of these jumps, obtained averaging these compositions and jumps along "wide" volumes and "long" times, it is the so called diffusion phenomenon. High temperatures means a lot of jumps and a faster diffusion rate of elements. The random walk method used in this model to diffuse elements through the microstructure accounts only for these atomic jumps [7], not taking into account concentration gradients or any other macroscopic variables. A Monte Carlo Step (MCS) will be defined when all the atoms of the simulated system try a single jump each one. As an example to clarify this, if a simulated system has a million atoms and we try ten millions jumps we had simulate an arbitrary time of 10 MCS. Proposed model distinguishes between two kind of atoms from the diffusional point of view; big atoms that occupy substitutional sites (Fe and $\mathrm{V}$ ) and small atoms that occupy octahedrical interstitial sites $(\mathrm{C}$ and $\mathrm{N})$.

The big elements, Fe and $\mathrm{V}$, are located in the substitutional sites of the lattice. The swap between two atoms of this kind requires a lot of energy and it is very difficult to take place. The diffusion of these atoms is usually controlled by the movement of vacancies. It is possible to calculate the concentration of vacancies for the austenitic matrix by using the Eq. 1 [8].

$$
C_{v}^{e q}(T)=K_{v} \exp \left(\frac{\Delta H_{v}^{f}}{k_{b} T}\right)
$$


where $C_{v}{ }^{e q}$ is the fractional concentration of vacancies present in the material, $K_{v}$ is a dimensionless pre-exponential factor that ranges from 2 to $10, \Delta H^{f}{ }_{v}$ is the vacancy formation enthalpy and its value is $1.4 \mathrm{eV}$ for the austenite, the $k_{b}$ is the Boltzman constant and $T$ is the absolute temperature. For the austenite at $1108 \mathrm{~K}$, the resulting vacancy concentration is about $1.92 \cdot 10^{-6}$. For a system of $1 \times 1 \mu \mathrm{m}$ that contains more than 30 million of substitutional sites there are only 57 vacancies.

Some simulations were performed using proposed model to study the diffusion of a plain front of low V concentration in a Fe-V system, only allowing the vacancies movements. Similar simulations were also performed using the Dictra ${ }^{\mathrm{tm}}$ software, that solves the problem from a continuum point of view [9]. In order to match results from both, model and Dictra ${ }^{\mathrm{tm}}$, it is necessary to assume that $1.99 \cdot 10^{8} \mathrm{MCS}$ is equivalent to a second, for the case of the diffusion of vacancies.

Due to the small fraction of vacancies, and the huge number of jumps needed to diffuse the V, the model was modified to simulate its diffusion just by swapping the substitutional atoms, and forgetting the vacancies. With this new method to simulate one second diffusion there are only necessary $3.73 \cdot 10^{2} \mathrm{MCS}$, reducing the number of jumps in six orders of magnitude. Fig. 2 shows the $\mathrm{V}$ concentration profiles obtained moving vacancies, swapping substitutional atoms and solving continuum equations by Dictra ${ }^{\mathrm{tm}}$ for a system temperature of $1108 \mathrm{~K}$. From this figure it is possible to conclude that the results obtained by both proposed methods are indistinguishable. Due to the computational efficiency the second method is going to be used.

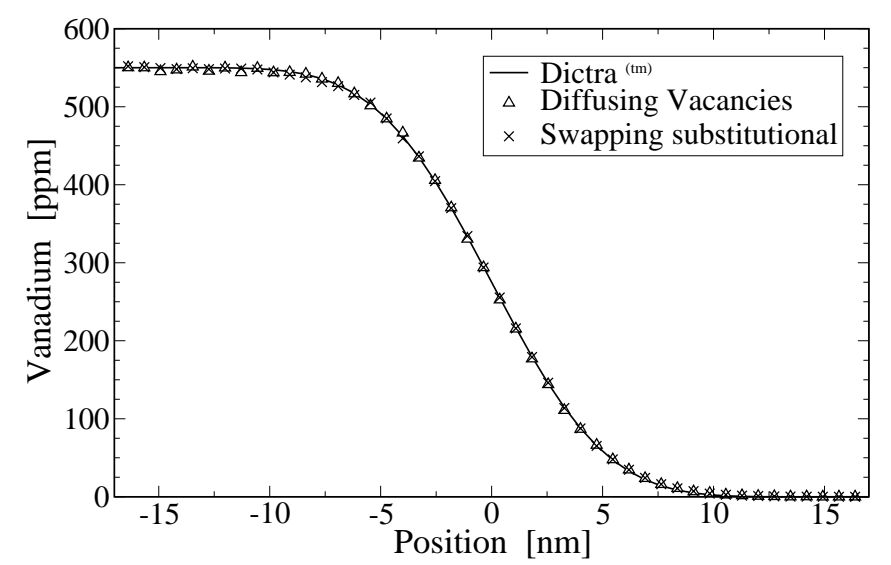

Figure 2: Concentration profiles of $\mathrm{V}$ after diffusing it during one second using the proposed model moving vacancies (triangles), swapping substitutional atoms (crosses) and solving continuum equations by software Dictra ${ }^{\mathrm{tm}}$ (solid line).

The diffusion of the small interstitial elements, such as $\mathrm{C}$ and $\mathrm{N}$, was simulated just allowing jumps from octahedrical to octahedrical sites. All octahedrical sites have the same probability to accept any interstitial atom, except for the case that the interstitial site is filled by an atom, in which case, the jump it is not allowed.

Similar simulations, as for the substitutional elements, were performed to study the relation between the diffusion rates obtained using proposed model and solving the continuum equations. For the case of the $\mathrm{C}$ and of the $\mathrm{N}$ there were necessary to perform $1.41 \cdot 10^{8} \mathrm{MCS}$ and $4.43 \cdot 10^{7}$ MCS respectively to simulate one real second.

Table 1 summarises the number of MCS needed to simulate one second on the diffusion for each element considered into the model and the possible jumps allowed for its diffusion. From this table it is clear that $\mathrm{V}$ diffusion is very slow compared with diffusion of interstitial elements. Due to this huge difference, the model is going to consider that the diffusion of $\mathrm{V}$ limits the microstructural evolution and that the $\mathrm{C}$ and $\mathrm{N}$ are able to diffuse infinitely fast, i.e. there are no macroscopic gradients in its composition and that the local concentration of these elements fluctuates a lot faster 
that the $\mathrm{V}$ concentration. These quick fluctuations could be made similar to a constant average concentration of $\mathrm{C}$ and $\mathrm{N}$ on simulated system voxels.

MCS needed to simulate the movement of atoms in one second.

\begin{tabular}{lcc}
\hline Element & MCS/second & Jumps allowed \\
\hline Vacancies & $1.99 \cdot 10^{8}$ & all substitutional sites \\
V & $3.73 \cdot 10^{2}$ & all substitutional sites \\
$\mathrm{C}$ & $1.41 \cdot 10^{8}$ & only free octahedrical sites \\
$\mathrm{N}$ & $4.43 \cdot 10^{7}$ & only free octahedrical sites \\
\hline
\end{tabular}

Table 1: Summary of the elements considered into the model, the MCS needed to diffuse them a second and the jumps allowed for each element.

Precipitation-dissolution. As previously explained, the state of a voxel could be determined as "matrix", "interface" or "precipitate". To simulate the precipitation-dissolution process it is necessary to control the flux of atoms between the precipitates and the matrix, via the interface voxels. Fig. 3 shows all possible kind of jumps that the model considers.

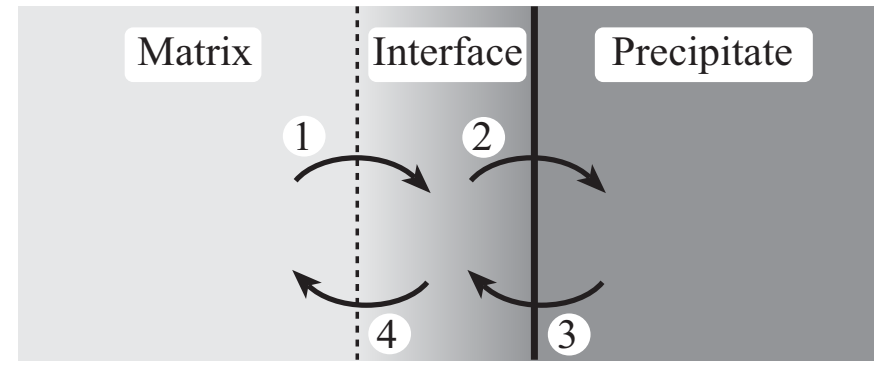

Figure 3: Different kinds of jumps that the model is able to take into account for simulating the precipitation-dissolution phenomenon.

The first kind of jump considered (1) is the movement of $\mathrm{V}$ atoms from matrix to interface voxels. This jump is allowed without restriction, but a prefixed fraction of atoms that make this jump sticks to the precipitate surface. As this fraction increases the precipitation phenomenon gets more important, increasing the size of particles. When an atom gets attached to the surface its internal state changes from "movable" to "fixed". The jump from the interface to the precipitate (2) it is also allowed to all atoms, but the state of them will always change to "fixed", as they become part of the precipitate.

To dissolve a precipitate it is necessary to move atoms from the precipitate to the matrix. In a first step, atoms have to jump from the precipitate to the interface (3). A predefined fraction of jumps of this kind are successful, and those successful jumps will change the state of those atoms into "movables", as they have been dissolved from the precipitate surface. Finally, the jump from the interface to the matrix voxels (4) is allowed for all "movable" atoms, but only a predefined fraction of "fixed" ones are able to leave the interface.

Changing the probabilities of these jumps it is possible to dissolve precipitates into the matrix, make them remain with the same volume fraction, or even make them to grow.

\section{Results and discussion}

The initial microstructures generated to check the behaviour of the proposed model, were generated using spherical seeds. The sizes of particles were that obtained by TEM measurement on a microalloyed steel [10]. Fig. 4 shows the cumulative distribution function (CDF) of empirically measured and model used sizes. 


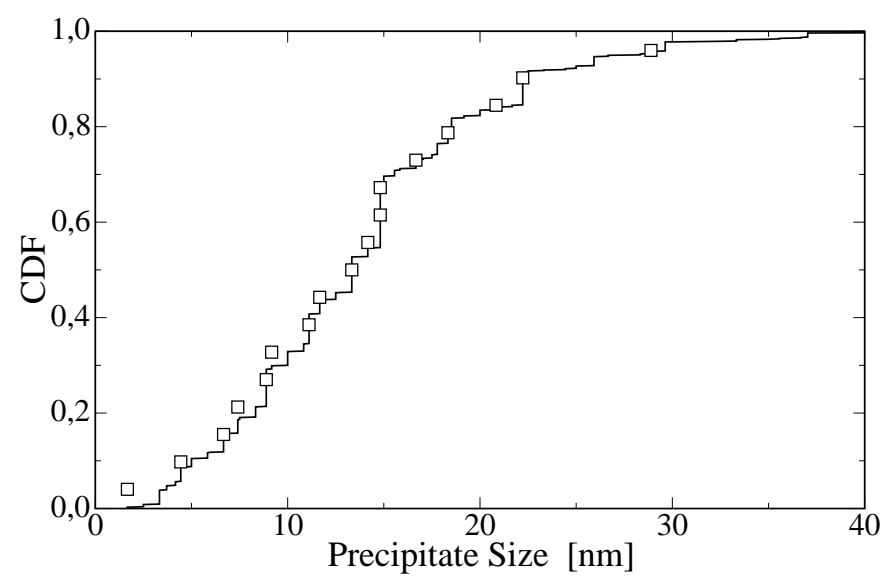

Figure 4: Cumulative distribution function of sizes empirically measured (solid line) and used by the model in present work (boxes).

In real steels it is very common to find areas with high concentration of precipitates and zones completely free of them, changing the average distance among them. This phenomenon even appears when the steel is previously homogenised during a long time. To simulate the behaviour of the model at these different zones, the particles, once their sizes were decided, are located at different size microstructures. As number and size of particles located in all microstructures are the same the volume fraction of precipitates will increase as the microstructure size decreases. The geometrical characteristics of microstructures used in this work are summarised in Table 2.

Geometrical characteristics of generated microstructures

\begin{tabular}{lccc}
\hline & Size $[\mathrm{nm} /$ side] & Size [voxels/side] & Total number voxels \\
\hline High vf. microstructure & 72.8 & 200 & $4.00 \cdot 10^{4}$ \\
Mid vf. microstructure & 294 & 808 & $6.53 \cdot 10^{5}$ \\
Low vf. microstructure & 1180 & 3240 & $1.05 \cdot 10^{7}$ \\
\hline
\end{tabular}

Table 2: Geometrical parameters that geometrically define the microstructures used to study the behaviour of the proposed model.

To locate the atoms in the microstructure, once the voxels are assigned to each matrix or to precipitates, it is necessary to know the composition of each phase. The initial data for the global composition of simulated microstructure was obtained again from empirical measurements. The equilibrium composition of both phases was obtained using MTData ${ }^{\mathrm{tm}}$ software [11]. Knowing these concentrations, the atoms could be assigned to the matrix or to the precipitate to fulfil the imposed concentration. As the composition of each phase is the same for all simulation, the microstructures with different volume fraction of precipitates will not have the same global composition. Table 3 summarises the compositions used for running the simulations in current work.

Composition of a real sample, pure phases, and simulated microstructures

\begin{tabular}{lccccc}
\hline & Fe & $\mathbf{V}$ & $\mathbf{C}$ & $\mathbf{N}$ & Vol.frac. C-N-V \\
\hline Real samples & 0.9814 & 0.00265 & 0.1568 & 0.00040 & - \\
Simulated matrix & 0.9606 & 0.00054 & 0.0136 & $4.127 \cdot 10^{-6}$ & - \\
Simulated precipitates & 0.0021 & 0.5077 & 0.3824 & 0.0962 & - \\
High vf. microstructure & 0.2959 & 0.3641 & 0.2686 & 0.07120 & 0.55377 \\
Mid. vf. microstructure & 0.9240 & 0.03294 & 0.03668 & 0.00633 & 0.03396 \\
Low vf. microstructure & 0.9814 & 0.00269 & 0.01549 & 0.00041 & 0.00211 \\
\hline
\end{tabular}

Table 3: Composition of a real sample of steel microalloyed with $\mathrm{V}$, composition computed by MTData $^{\mathrm{tm}}$ of pure phases at $1108 \mathrm{~K}$, and composition of simulated microstructures. 
Proposed model it is able to simulate various phenomena according to the values given to the parameters that determine the fraction of atoms that stick to, or released from, the precipitates surface. If the fraction of atoms attached to the surface is very small or the atoms "evaporation" fraction is very big, the precipitates will dissolve. Otherwise, if the fraction of attached atoms is very big, the precipitate will grow. The equilibrium condition will be given when the attaching and releasing rates are equal. The conditions to get these behaviours, not only as function of attaching and releasing rates but as function of $\mathrm{V}$ concentration present in both phases are given by Eq. 2 .

$$
\begin{aligned}
& F_{V \text { prec }} \cdot R_{r e l}>F_{V m t r x} \cdot R_{\text {stck }} \text { (dissolution) } \\
& F_{V \text { prec }} \cdot R_{\text {rel }} \approx F_{V m t r x} \cdot R_{\text {stck }} \text { (equilibrium) } \\
& F_{V \text { prec }} \cdot R_{r e l}<F_{V m t r x} \cdot R_{\text {stck }} \text { (precipitation) }
\end{aligned}
$$

where $F_{V \text { prec }}$ and $F_{V m t r x}$ are the fraction of substitutional sites filled by $\mathrm{V}$ atoms in the precipitates and in the matrix respectively, $R_{\text {rel }}$ probability of releasing an atom from a precipitate and $R_{\text {stck }}$ is the probability of sticking an atom to the surface of a precipitate.

The equilibrium conditions used in this work were $(0.0001685,0.3)$. Using these conditions, the volume fraction of precipitates does not change, but there is a flux of $\mathrm{V}$ atoms towards and from the precipitate. As both fluxes have the same average value, the net flux trough the interface is zero. The average number of atoms that cross the interface during simulations are $259 \mathrm{~V}$ atoms $/ \mathrm{s}$, and the number of stick (or released) atoms per time unit are $104 \mathrm{~V}$ atoms/s. From these data it is possible to deduce that the system is in a dynamic equilibrium.

From empirical measurements it is possible to know the real evolution of particle sizes during ten minutes of heat treatment at $1108 \mathrm{~K} \mathrm{[10]}$. By the other side it is possible to simulate the final microstructure that will appear after a ten-minutes simulation. Fig. 5 shows the cumulative distribution function for real and for simulated sizes.

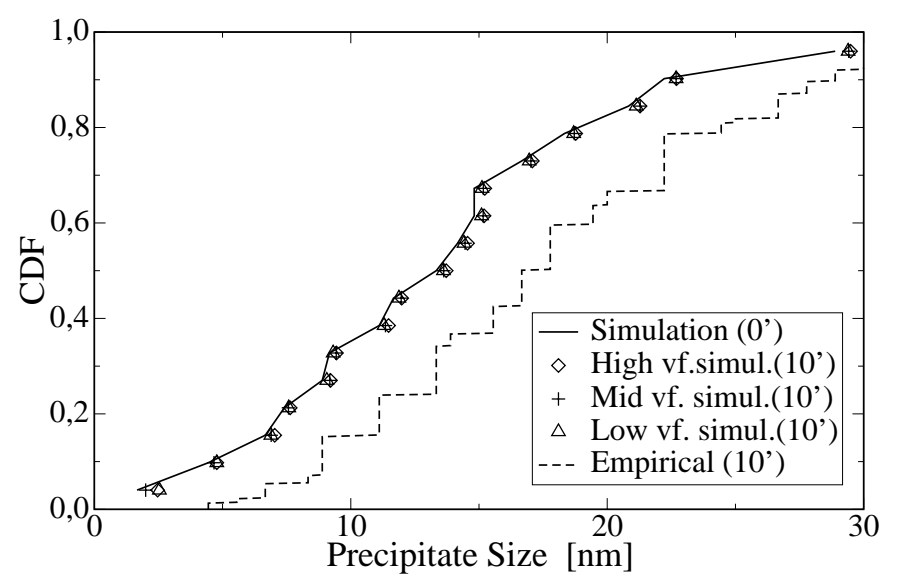

Figure 5: Cumulative distribution function of sizes empirically measured (dashed line) and obtained by proposed model (symbols) after a ten-minutes treatment at $1108 \mathrm{~K}$.

From this figure it is possible to deduce that there are no a clear influence of the distance between particles and the evolution of precipitates sizes, at least for this simulated time. On the other hand it is clear that the increase on average size predicted from the model is not in agreement with real measurements. The model predicts an increase of $1.6 \%$ in the average size and the empirical measurements show an increase of a $20 \%$. This difference is consequence of several factors, such as the homogenous composition of phases at the first stage of the simulation or the 2D nature of the model. 


\section{Conclusions}

The proposed model, which takes into account local fluctuations of concentration, is able to simulate qualitatively the fact of dissolution and grow of $\mathrm{C}-\mathrm{N}-\mathrm{V}$ precipitates.

In the case of the dynamic equilibrium model is able to predict an increase on the average precipitate size, but very slowly in comparison with real measurements.

Further work has to be performed to extend the proposed model to 3D to try to match empirical measurements with simulated ones.

\section{Acknowledgments}

Authors would like to thank the Spanish Ministry of Science and Technology (MCyT) for the financial support. J. Aldazabal thanks for the sponsorship under the project MAT2003-04314 and C. Garcia-Mateo for the financial support in the form of a Ramón y Cajal contract (Programa RyC 2004).

\section{Bibliography}

[1] M.F. Ashby, D.R.H. Jones: Engineering Materials 1: An introduction to their Properties and Applications (Ed. Butterworth-Heinemann Ltd, Cambridge 1980) p. 97-102.

[2] T. Gladman: The Physical Metallurgy of Microalloyed Steels (The Institute of Materials, 1997) p. 34.

[3] J. Geise and Ch. Herzig: Z. Metallkd. Vol.78 (1987), p.291

[4] J. Agren: Scripta Met. Vol.20 (1986), p.1507-1510

[5] D. Raabe: Computational Material Science: the simulation of materials, microstructures and properties (Ed. Wiley-VCH, 1998) p. 102-104.

[6] R.W. Cahn, P. Haasen: Physical Metallurgy (vol.2) (Ed. Elsevier Science, Netherlands 1996) p. 1561-1569.

[7] P.G. Shewmon: Diffusion in Solids (Ed. McGraw-Hill, 1963) p. 47-51.

[8] M.E. Glicksman: Diffusion of solids: field theory, solid-state principles, and applications (Ed. John Wiley \& Sons, Inc., New York 2000) p. 228-233.

[9] Dictra User's Guide (Dept. of Materials Science and Engineering, Royal institute of Technology, Sweden 1998) p. 63-71.

[10] Garcia-Mateo, $C$. Influencia de la aleación con $V$ en el conformado en tibio de aceros de contenido medio/alto en $C(\mathrm{PhD}$. Thesis, University of Navarra, San Sebastian 2000) p.4.464.49 .

[11] MT-DATA: Phase diagram Calculation software (National Physical Laboratory, Teddington, UK, 2003). 